\title{
Tunable CMOS Delay Gate with Reduced Impact of Fabrication Mismatch on Timing Parameters
}

\author{
Przemyslaw Mroszczyk and Piotr Dudek \\ School of Electrical \& Electronic Engineering \\ The University of Manchester \\ Manchester, M13 9PL, United Kingdom \\ przemyslaw.mroszczyk@postgrad.manchester.ac.uk \\ p.dudek@manchester.ac.uk
}

\begin{abstract}
This paper presents the analysis and design of a simple one-stage tunable delay gate with improved matching properties as compared with the commonly used "current starved inverter". The operation of two delay lines employing these structures in a standard $90 \mathrm{~nm}$ CMOS technology was verified based on the post layout mismatch Monte Carlo simulations. Accounting for the fabrication mismatch, the delay generated by the proposed "output-split inverter" (OSI) circuit is about $10-30 \%$ more accurate as compared to the conventional current starved inverter occupying the same chip area.
\end{abstract}

\section{INTRODUCTION}

An inherent shortcoming of any VLSI fabrication process is a certain degree of random variability of all the physical parameters of the manufactured devices, mainly MOS transistors used in any standard CMOS technology. Usually, the nature of these variations can be seen as a global parameter fluctuation, with a long distance correlation, and a local one, randomly affecting the parameters of equally designed and closely laid out devices [1]. Especially local variations, known as fabrication mismatch, are more difficult to mitigate and may significantly degrade the performance of a circuit. A well known method for mismatch optimization is transistor size scaling based on the assumption that the local variations average out as the MOS device area increases [2]. Proper layout drawing techniques (accounting for the proximity effects, the STI stress, the metal coverage, the distancedependent parameter variability etc.) and some circuit specific design techniques such as offset compensation, bias point optimization, design redundancy and averaging, and post fabrication trimming could be applied to reduce mismatch and increase the fabrication yield [3]. Usually the efficient mismatch optimization of a larger analogue or mixed system is difficult and requires specific simulation tools. In many cases, the correct operation of such a system relies mainly on the correct operation of its critical sub-blocks mostly affecting its total performance, and these circuits should be optimized in the first instance [4].

The functional sub-block considered in this paper is a tunable delay gate used in a number of applications requiring the generation of controlled delay time intervals such as Delay Locked Loops [5], time-to-digital converters [6], silicon pixel readout circuits for particle detection [7]-[9] and asynchronous processor arrays [4], where the mismatch in time intervals generated by different delay gates or delay lines is reported as the dominant factor limiting the precise operation of the whole circuit. The applications found in literature employ a typical structure of a delay gate based on the current starved inverter (CSI) shown in Fig. 1a, where the mismatch reduction is usually achieved by the proper scaling (enlarging) of all the transistors or just the current controlling one $\left(M_{l}\right)$ [5], [8].
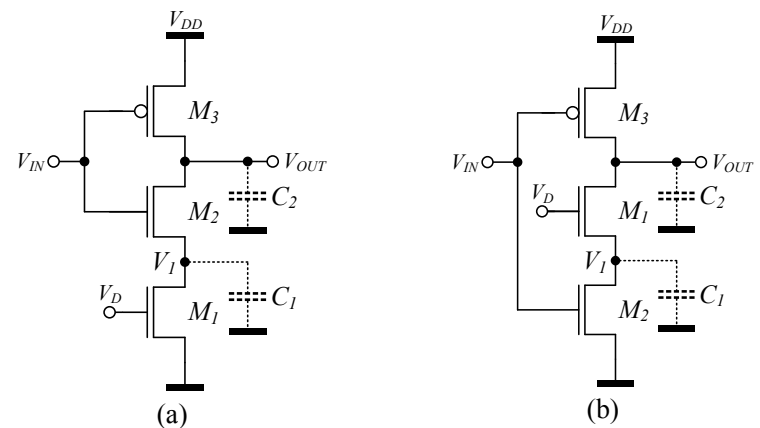

Figure 1. Schematic diagrams of the delay gates: a) the commonly used current starved inverter (CSI), b) the proposed "output-split inverter" (OSI)

In this paper an alternative structure of a delay gate, the "output-split inverter" (OSI) presented in Fig. 1b, is analysed. The only difference is the location of the current controlling transistor $M_{l}$ on the drain rather than source side of $M_{2}$, which separates or "splits" the output of the inverter. It is shown that this structure has less influence of MOS parameter mismatch on the generated delay variability than the CSI gate when both of them occupy the same area. The proposed OSI structure was also used in the build of a charge pump circuit [5] and a linearly tuned delay element [10]. Nevertheless, to the best of our knowledge, no previous work, except our applicationoriented paper [4] postulate the superior matching performance of this circuit. Section II of this paper analyses the operation of both gates, Section III explains the influence of the MOS fabrication mismatch on the delay variability, 
Section IV presents and discusses the simulation results and Section V concludes the paper.

\section{CIRCUIT ANALYSIS}

The operation of both delay gates presented in Fig. 1 is similar. Transistors $M_{2}$ and $M_{3}$ operate as switches determining the direction of the current flow (as in a static CMOS inverter) and additionally, for the rising edge of $V_{I N}$, $M_{2}$ turns on and $M_{1}$ limits the current discharging the output load capacitance depending on $V_{D}$. However, the simulation results of these gates reveal substantial differences in their operation (Fig. 2) which are of high importance in terms of the device matching properties (the details of the simulations are presented in Section IV). In the following we provide a simplified analysis presenting only the first-order behaviour addressing the major differences between circuits from Fig. 1.

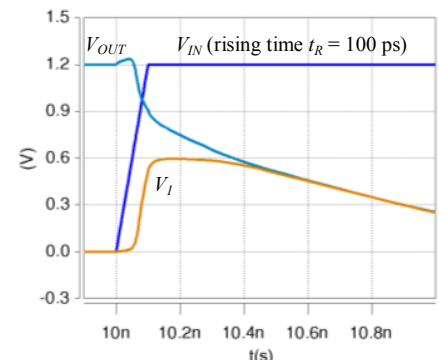

(a)

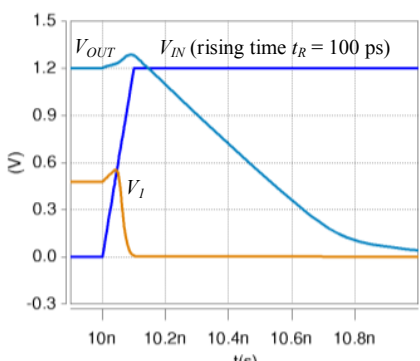

(b)
Figure 2. Transient analysis of the circuits from Fig. 1 for the rising edge of the input volage $V_{I N}\left(W_{l} / L_{l}=1 \mu \mathrm{m} / 1 \mu \mathrm{m}, W_{2,3} / L_{2,3}=1 \mu \mathrm{m} / 90 \mathrm{~nm}, C_{2}=1 \mathrm{fF}\right.$, $C_{l}=0$ - only inherent source/drain MOS capacitances assumed, $V_{D D}=1.2 \mathrm{~V}$, $t_{R}=100 \mathrm{ps}$ ): a) CSI gate for $V_{D}=450 \mathrm{mV}, \mathrm{b}$ ) OSI gate for $V_{D}=500 \mathrm{mV}$.

\section{A. Current Starved Inverter (CSI)}

A simplified analysis of the CSI circuit showing the transitions of voltages $V_{I N}, V_{O U T}$ and $V_{l}$ is presented in Fig. 3. There are three phases on the timeline: the initial phase $\left(t<t_{1}\right)$, the switching phase $\left(t_{1}<t<t_{2}\right)$ and the discharge phase $\left(t>t_{2}\right)$.

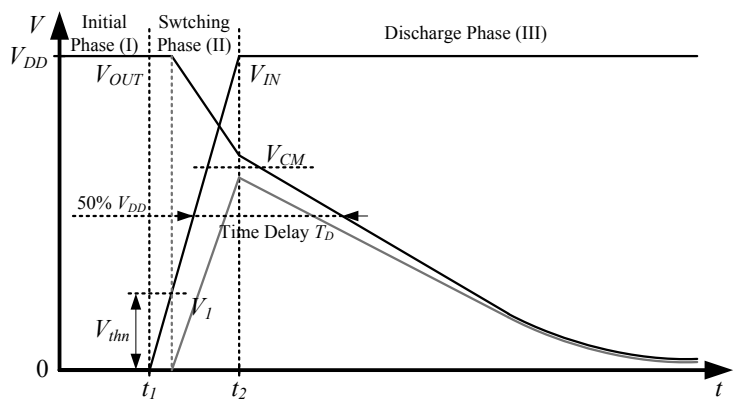

Figure 3. The behaviour of the CSI circuit in a transient state.

In the initial phase, the capacitance $C_{1}$ is discharged to zero through $M_{1}$ (for $V_{D}>0$ ) and the capacitance $C_{2}$ is charged to $V_{D D}$ through $M_{3}\left(V_{I N}=0\right)$. In the switching phase, the rising edge of $V_{I N}$ simultaneously turns $M_{2}$ on and turns $M_{3}$ off. Due to the charge sharing between $C_{1}$ and $C_{2}$, and the drain current of $M_{3}$, the capacitance $C_{2}$ gradually discharges and $C_{1}$ charges to a higher voltage. As a result $V_{O U T}$ and $V_{I}$ converge closer to the common level $V_{C M} \approx V_{D D} \times C_{2} /\left(C_{1}+C_{2}\right)$ denoting the starting point for the discharge phase where $M_{2}$ (for $V_{I N}=V_{D D}$ ) keeps voltages $V_{\text {OUT }}$ and $V_{1}$ almost equal. The discharge rate of $C_{1}$ and $C_{2}$ depends mostly on the drain current of $M_{1}$ controlled by $V_{D}$. The proposed analysis does not consider the variable signal slopes and the charge injection effects of $M_{2}$ and $M_{3}$. These issues are covered in Section IV when simulating delay lines.

\section{B. Output-Split Inverter (OSI)}

The analysis of the OSI circuit showing the transitions of voltages $V_{I N}, V_{O U T}$ and $V_{l}$ is presented in Fig. 4.

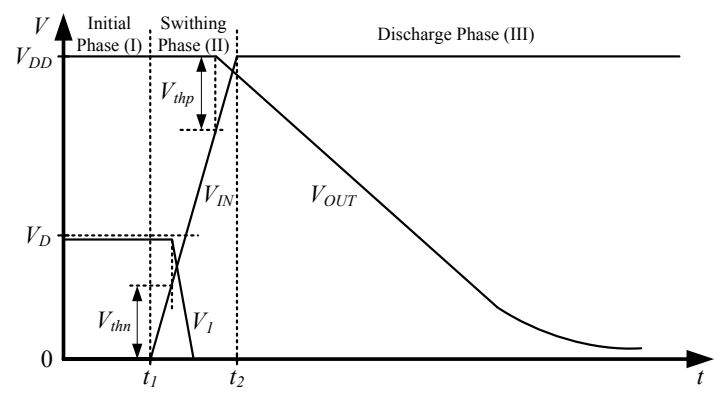

Figure 4. The behaviour of the OSI circuit in a transient state.

In the initial phase the capacitance $C_{2}$ remains charged to $V_{D D}$ through $M_{3}$ and the capacitance $C_{1}$ remains charged closely to $V_{D}$ through $M_{l}$ operating in the weak inversion and conducting the off leakage current of $M_{2}\left(V_{I N}=0\right)$. In the switching phase the rising edge of $V_{I N}$ turns $M_{2}$ on (when $V_{I N}>$ $\left.V_{t h n}\right)$, which quickly discharges $C_{1}$ to zero and, after that, turns $M_{3}$ off (when $V_{I N}>V_{D D}-\left|V_{t h p}\right|$ ) and $C_{2}$ starts discharging with a rate dependent mainly on the drain current of $M_{l}$ controlled by $V_{D}$. The further operation of this gate is practically the same as in the case of the CSI one.

\section{MISMATCH ANALYSIS}

In literature the mismatch analysis and optimization of the timing parameters of the current starved inverter is usually done assuming that the transistors $M_{2}$ and $M_{3}$ (Fig. 1a) work as ideal switches and the precision of the generated time delay $T_{D}$ depends mainly on the current limiting transistor $M_{l}$ [5]. More elaborate analysis of this circuit, accounting for the inter- and intra- die parameter fluctuations (also in switching transistors), was presented in [8]. The majority of works conclude that the precision of the time $T_{D}$ could easily be improved by enlarging the current limiting transistor but the corresponding precisionarea trade off should also be considered. In this section we focus on the influence of the MOS parameter variability on the signal transitions in the switching phase and show its impact on the starting point of the discharge phase in both circuits.

\section{A. Mismatch in the CSI circuit}

The analysis of the CSI circuit showing the influence of the MOS parameter fluctuations on the variability of the $V_{C M}$ voltage and the generated delay time $T_{D}$ is presented in Fig. 5. The common level $V_{C M} \approx V_{D D} \times C_{2} /\left(C_{1}+C_{2}\right)$ will also depend on slightly different parameters of all the MOS transistors resulting from the fabrication mismatch. For example, due to the random variation of the threshold voltage $V_{t h n}$ of $M_{1}$ this transistor may be slightly "stronger" (for lower values of $V_{t h n}$ ) 
or slightly "weaker" (for higher values of $V_{t h n}$ ) than a regular one. For the "stronger" $M_{l}$ the $V_{C M}$ voltage will be lower and the corresponding discharge slope will be steeper, whereas for the "weaker" $M_{1}$ the $V_{C M}$ voltage will reach a higher value and the discharge phase will take a longer time. As a result it can be observed that the variability of $V_{C M}$ voltage $\left(\Delta V_{C M}\right)$ affects the variability $\Delta T_{D}$ of the generated time delay $T_{D}$.

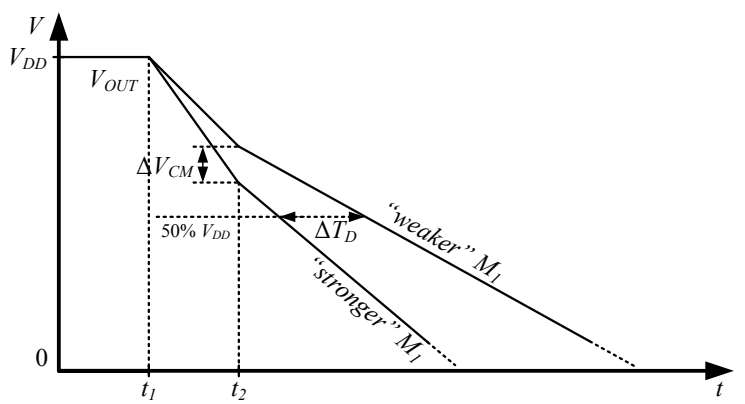

Figure 5. The transient state of the CSI circuit showing the influence of the MOS parameter variability on the $V_{C M}$ voltage and the generated delay $T_{D}$.

\section{B. Mismatch in the OSI circuit}

The analysis of the OSI circuit showing the influence of the MOS parameter fluctuations on the variability of the generated time delay $T_{D}$ is presented in Fig. 6. Due to the current limiting transistor $M_{l}$ "splitting" the output of the inverting stage, the rising edge of $V_{I N}$ may not force an immediate transition of $V_{\text {OUT }}$. While the capacitance $C_{I}$ quickly discharges to zero (Fig. 4), $M_{3}$ still pulls the output node up to $V_{D D}$ postponing the discharge phase roughly until $V_{I N}>V_{D D}-\left|V_{t h p}\right|$. For the slightly "stronger" transistor $M_{3}$ with lower threshold voltage $\left|V_{t h p}\right|$ the discharge phase may begin later than for the slightly "weaker" one. Additionally, the slightly "stronger" transistor $M_{1}$ may discharge $C_{2}$ faster also increasing the variability of the generated delay time. The influence of the first factor $\left(\Delta V_{t h p}\right)$ is usually suppressed by a sharp slope of $V_{I N}$, whereas in case of the second one the discharge phase always begins for the fully charged $C_{2}$. Because of this the starting point of the discharge phase is more stable and the discharge time of $C_{2}$ is longer for the same current of $M_{1}\left(C_{2}\right.$ charged to $\left.V_{D D}>V_{C M}\right)$ as compared to the CSI structure, which makes the generated delay time of the OSI circuit less prone to fabrication mismatch.

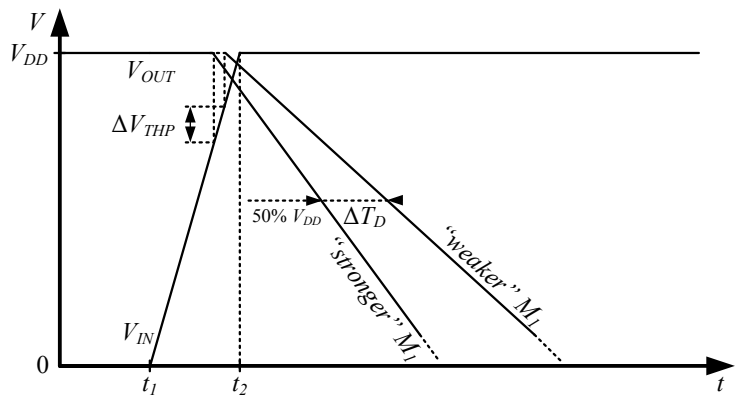

Figure 6. The transient state of the OSI circuit showing the influence of the MOS parameter variability on the generated delay time $T_{D}$.

\section{Simulation RESUltS AND DiscUSSION}

The operation of both circuits (Fig. 1) was verified using HSPICE and mismatch Monte Carlo MOS transistor models from a standard $90 \mathrm{~nm}$ CMOS technology. We assumed fixed load $C_{2}=1 \mathrm{fF}$ corresponding to a typical load capacitance in this technology and considered only the size-dependent source /drain junction capacitances of MOS transistors $\left(C_{l}=0\right)$. The rising time of the input signal $t_{R}=100 \mathrm{ps}$ (not critical in terms of the qualitative analysis) and $V_{D D}=1.2 \mathrm{~V}$.

(a)
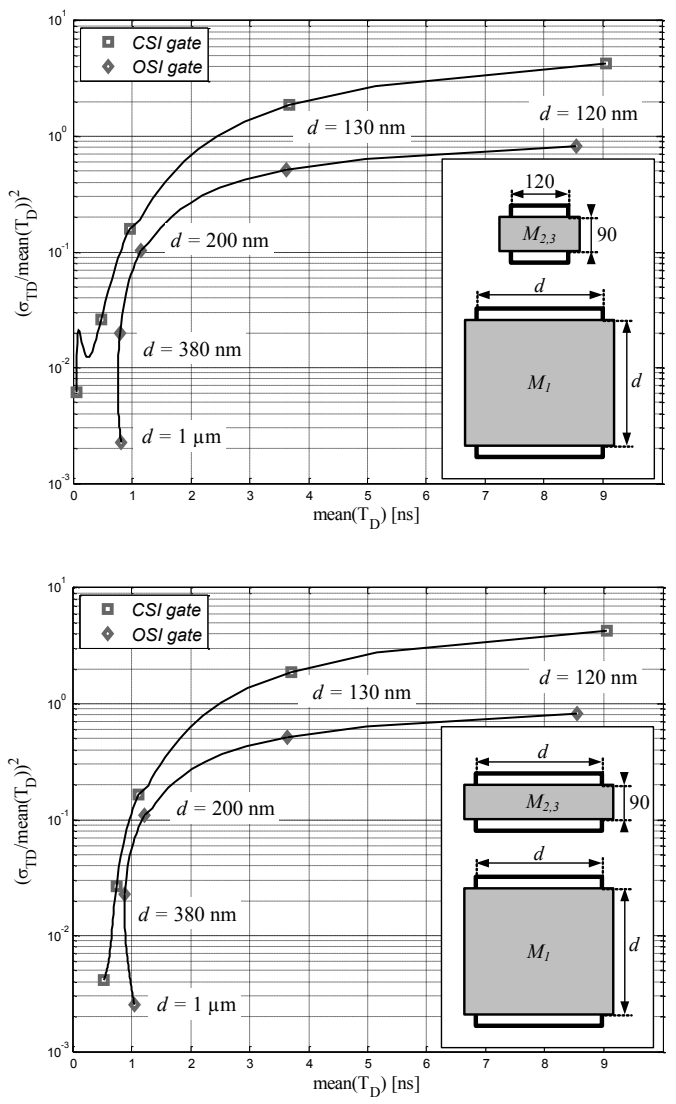

Figure 7. The normalised delay variance in terms of the mean $T_{D}$ of the CSI and OSI circuits for two different scaling approaches where: a) only the current limiting transistor $M_{1}$ is scaled, b) all the transistors are scaled.

The statistical parameters of both structures using two different approaches to transistor scaling, where only the current limiting transistor $M_{1}$ is scaled $\left(W_{l}=L_{1}=d, W_{2,3}=\right.$ $120 \mathrm{~nm}, L_{2,3}=90 \mathrm{~nm}$ ) and when widths of the switching transistors $M_{2,3}$ are also scaled simultaneously with $M_{1}\left(W_{1,2,3}\right.$ $=L_{l}=d, L_{2,3}=90 \mathrm{~nm}$ ), where $d$ is swept between $120 \mathrm{~nm}$ and $1 \mu \mathrm{m}$, were obtained from 500 Monte Carlo runs for each case. The corresponding results showing the normalized delay variance [9] in terms of the mean value of the delay time for the CSI and the OSI gates (for $V_{D}$ fixed to $420 \mathrm{mV}$ ) are presented in Fig 7 . In both scaling schemes, the gate of $M_{l}$ is square in order to reduce the variability of its width and length and increase its active area and hence reduce the parameter mismatch [2]. It can be observed that the OSI structure exhibits less delay time variability than the CSI variant for the same area (e.g. for $W_{l} / L_{l}=1 \mu \mathrm{m} / 1 \mu \mathrm{m}$ in Fig. $7 \mathrm{~b}$ the accuracy of the OSI circuit is about $20 \%$ higher than for the CSI one). 
In order to compare the operation of both gates accounting for the variability in the switching levels, signal slopes and load capacitances, models of two delay lines consisting of 16 serially connected symmetric (with two complementary current limiting transistors) CSI and OSI gates were designed for $W_{1,4} / L_{1,4}=1 \mu \mathrm{m} / 0.5 \mu \mathrm{m}$ and $W_{2,3} / L_{2,3}=1 \mu \mathrm{m} / 90 \mathrm{~nm}$ (Fig. 8).

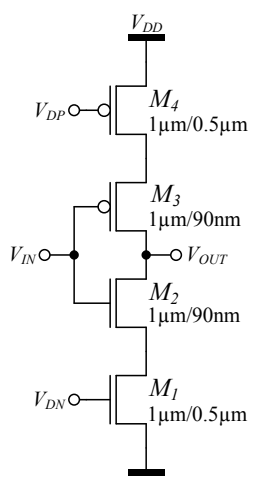

(a)

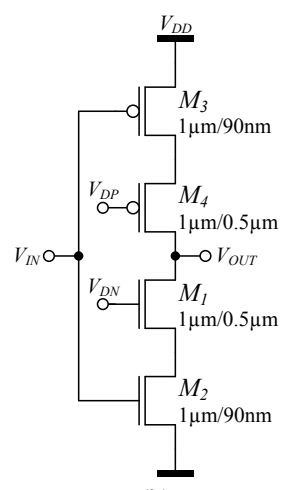

(b)
Figure 8. Schematic diagrams of the delay gates with two complementary current limitting transistors $M_{l}$ and $M_{4}$ : a) CSI variant, b) OSI variant.

The layouts of both delay lines designed in a standard $90 \mathrm{~nm}$ CMOS technology are presented in Fig. 9. Fig. 10 shows the schematic diagram of the test circuit used for the simulations. The two additional inverters (with transistors of $W / L=1 \mu \mathrm{m} / 90 \mathrm{~nm}$ ) on the input and the output of the delay line were added to assure more realistic models of the input signal source and the output load.

(a)

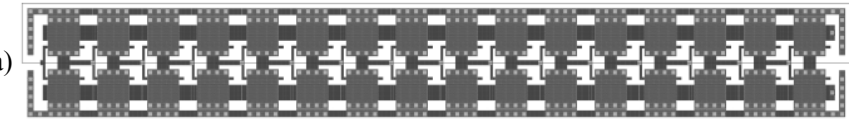

(b)

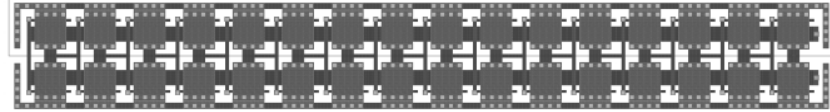

Figure 9. Layouts of the delay lines consisting of 16 serially connected a) CSI gates and b) 16 OSI gates (size of one line: $3.7 \mu \mathrm{m} \times 27 \mu \mathrm{m}$ ).

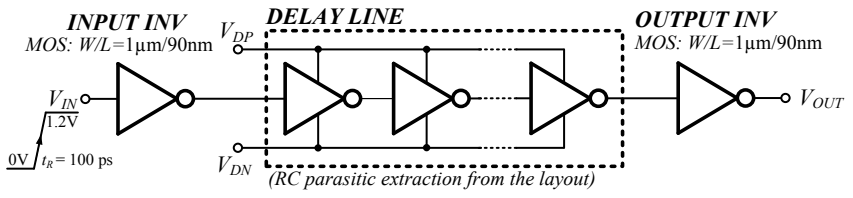

Figure 10. Schematic diagram of the test circuit used in the simulations.

The results of the Monte Carlo mismatch simulations of the test circuits with the post layout RC extracted models of the delay lines (also accounting for the mismatch in MOS transistors in the additional inverters) are presented in Fig. 11. The figure shows the normalized variance of the generated delay time for different bias voltages $\left(V_{D N}=400 \ldots 900 \mathrm{mV}\right.$ and $\left.V_{D P}=V_{D D}-V_{D N}=800 \ldots 300 \mathrm{mV}\right)$ in terms of the mean value of $T_{D}$. The simulation results (verified across all PVT corners) show that the use of the OSI gates in the design of delay lines improves the precision of the generated delay time $T_{D}$ (compared to the CSI variant of the same size) not requiring any additional transistor scaling or area increase.

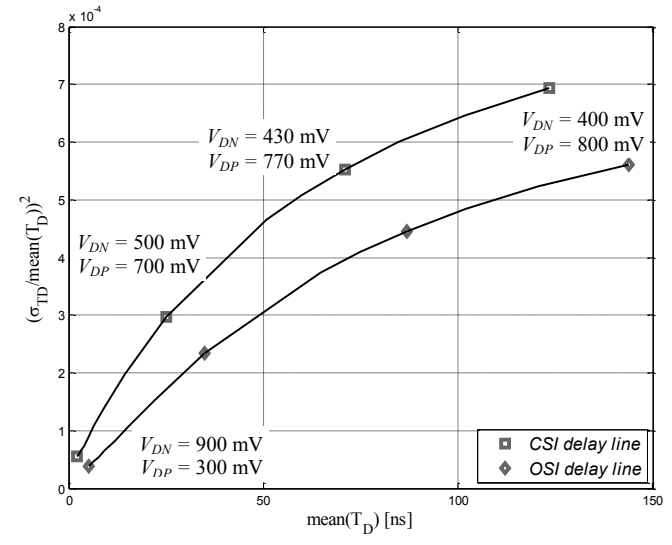

Figure 11. The normalized delay variance in terms of the mean $T_{D}$ generated by the CSI and OSI delay lines for $V_{D N}=400 \ldots 900 \mathrm{mV}$ and $V_{D P}=V_{D D}-V_{D N}$.

\section{CONCLUSIONS}

This paper presents the design and the analysis of the tunable delay gate based on the "output-split inverter" (OSI). The proposed structure operates with a higher precision than the commonly used current starved inverter (CSI) circuit in the presence of fabrication mismatch. The operation and timing parameters of both structures were verified based on the mismatch Monte Carlo simulations of the post layout models of delay lines designed in a standard $90 \mathrm{~nm}$ CMOS technology. In typical applications the proposed OSI structure may show about $10-30 \%$ higher accuracy than its CSI counterpart when occupying the same chip area.

\section{REFERENCES}

[1] Pelgrom M., Duinmaijer A. C., Welbers A., "Matching Properties of MOS Transistors", IEEE Journal of Solid-State Circuits, vol. 24, no. 5, pp. 1433-1439, Oct. 1989.

[2] Lovett S., Welten M., Mathewson A., Mason B., "Optimising MOS Transistor Mismatch", IEEE Journal of Solid-State Circuits, vol. 33, no. 1, pp. 147-150, Jan. 1998.

[3] Kinget P., "Device Mismatch and Tradeoffs in the Design of Analog Circuits", IEEE Journal of Solid-State Circuits, vol. 40, no. 6, pp. 12121224, Jun. 2005

[4] Mroszczyk P., Dudek P., "Trigger-Wave Collision Detecting Asynchronous Cellular Logic Array for Fast Image Skeletonization", ISCAS 2012, May 2012.

[5] Christiansen J., "An Integrated CMOS 0.15ns Digital Timing Generator for TDC's and Clock Distributed Systems", IEEE Trans. on Nuclear Science, vol. 42, no. 4, pp. 753-757, Aug. 1995.

[6] Dudek P., Szczepanski S., Hatfield J., "A High-Resolution CMOS Time-to-Digital Converter Utilizing a Vernier Delay Line", IEEE Trans. on Solid-State Circuits, vol. 35, no. 2, pp. 240-247, Feb. 2000.

[7] Heijne E., et al., "LHC1: A semiconductor pixel detector readout chip with internal, tunable delay providing a binary pattern of selected events", Nuclear Instruments and Methods in Physics Research, Section A 383, pp. 55-63, Elsevier, 1996.

[8] Cantatore E., et al., "Statistical analysis and optimisation of delay line chains for pixel readout electronics", Nuclear Instruments and Methods in Physics Research, Section A 395, pp. 318-323, Elsevier, 1997.

[9] Bolt M., et al., "Matching Properties of MOS Transistors and Delay Line Chains with Self-Aligned Source/Drain Contacts", Proc. of the IEEE Int. Conference on Microelectronic Test Structures, Mar. 1996.

[10] Jovanovic G., Stojcev M., "Linear Current Starved Delay Element", XL Int. Scientific Conference on Information Communication and Energy Systems and Technologies (ICEST), Jun. 2005. 